\title{
A three-factor model of syllogistic reasoning: The study of isolable stages
}

\author{
DONALD L. FISHER \\ University of Michigan, Ann Arbor, Michigan 48109
}

\begin{abstract}
Computer models of the syllogistic reasoning process are constructed. The models are used to determine the influence of three factors-the misinterpretation of the premises, the limited capacity of working memory, and the operation of the deductive strategy-on subjects' behavior. Evidence from Experiments 1, 2, and 3 suggests that all three factors play important roles in the production of errors when "possibly true" and "necessarily false" are the two response categories. This conclusion does not agree with earlier analyses that had singled out one particular factor as crucial. Evidence from Experiment 4 suggests that the influence of the first two factors remains strong when "necessarily true" is used as an additional response category. However, the third factor appears to interact with task demands. Some concluding analyses suggest that the models offer alternative explanations for certain well established results.
\end{abstract}

One common finding has emerged from the many different studies of categorical syllogisms. The studies all report that subjects frequently derive conclusions to the syllogisms that are logically incorrect. Chapman and Chapman (1959), Erickson (1974), and Revlis (1975a, $1975 \mathrm{~b})$ argue that the problem lies with the interpretation or encoding of the premises of the syllogism. Guyote and Sternberg (Note 1) and Stemberg and Turner (Note 2) conclude that the information processing demands of the task are an important determinant of the errors subjects make. Ceraso and Provitera (1971), Erickson (1978), and Johnson-Laird and Steedman (1978) argue that the deductive or problem solving strategy is itself an error-laden process. These three factors have been analyzed separately and in pairs, but never all in concert. One purpose of the present studies was to construct a model that could identify the relative contribution of each of the above three factors-premise interpretation, information processing capacity, and deductive strategy-to the production of incorrect responses.

A number of factors in addition to those mentioned above have been found to influence subjects' performance. It has been argued that the figure of the syllogism can affect subjects' behavior (Dickstein, 1978a; Frase, 1968; Johnson-Laird \& Steedman, 1978; Roberge, 1971). It has also been argued that the performance of

These experiments were carried out as part of a research requirement of the $\mathrm{PhD}$ program in psychology at the University of Michigan. Portions of this paper were presented at the meetings of the Society for Mathematical Psychology, August 1979. Clyde Coombs, Marilyn Shatz, and Susan Duffy kindly reviewed various portions of the manuscript. Special thanks go to Keith Holyoak (who suffered through three drafts of the manuscript) and Robert Sternberg at Yale University. I am indebted to each of the above individuals for their helpful comments and criticisms. subjects on syllogisms with concrete premises (e.g., "All horses are animals") will differ from their performance on abstract syllogisms (e.g., "All A are B") (Revlis, 1975a; Wilkins, 1928). And it has been argued that the performance of subjects on syllogisms with emotional premises (e.g., "All whites in Shortmeadow are welfare recipients") will differ from their performance on syllogisms with neutral premises (Revlin \& Leirer, 1978). This list by no means exhausts the additional factors that have been identified as important. But the list does serve to indicate the rather limited scope of the new models proposed in this paper. The models to be constructed are confined to the explanation of subjects' behavior when subjects are given syllogisms with neutral, abstract premises.

It will be necessary throughout the paper to refer to several different types of set relations: equivalence, subset-superset, overlap, and disjoint. These set relations can be represented as Euler diagrams:
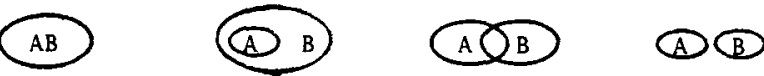

From left to right, the Euler diagrams represent the equivalence relation ( $A$ and $B$ are equivalent sets), the subset-superset relation (A is a proper subset of $B$; $B$ is a proper superset of $A$ ), the overlap relation (at least one $A$ is a $B$; at least one $A$ is not $a B$; at least one $B$ is not an $A$ ), and the disjoint relation ( $A$ and $B$ are disjoint sets).

Various terms are used throughout the paper that are best introduced at this point. A syllogism consists of a major premise (e.g., "All A are B") and a minor premise (e.g., "All B are C"). The major premise describes a relation between the predicate ("A") and the middle 
term ("B"), and the minor premise describes a relation between the subject (" $\mathrm{C}$ ") and the middle term (again "B"). One or more conclusions (e.g., "All C are A") follow the syllogism. The conclusion relates the subject and predicate to each other. Each syllogism can exist in any one of four figures (the figure is determined by the order of the predicate and middle term in the major premise and the order of the subject and middle term in the minor premise).

Before proceeding with the discussion of individual experiments, an overview of the paper is in order. The syllogistic reasoning process can be represented as a series of stages, the output from one stage providing the input to another stage. In general, the stages of complex cognitive processes cannot be studied in isolation from each other. However, this is not the case with syllogistic reasoning. In particular, it is possible to provide subjects with the theoretical inputs to a single stage within a given model and to have subjects produce or respond to the theoretical outputs from that stage. Such an approach characterizes both Experiments 1 and 3. That is, in Experiment 1 an early stage of the syllogistic reasoning process is examined in isolation, and in Experiment 3 a later stage is examined in isolation. The information gained from the study of the various isolable stages can then be used to analyze the relation between behavior in the complete syllogistic reasoning task and behavior on the tasks associated with the isolable stages. Experiments 2 and 4 focus on just this relation.

\section{PREMISE INTERPRETATION}

A subject's interpretation of the premises of a syllogism should determine in large measure which conclusions follow from the premises and which do not. For example, suppose a subject is given the following syllogism:

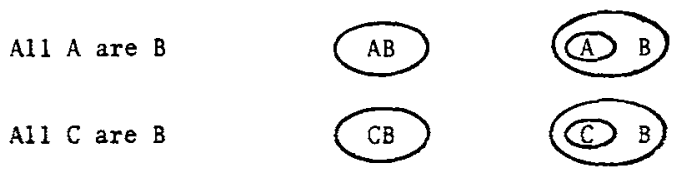

The Euler diagram representations of the first and second premises are listed opposite each premise. Note that the ideal or logical subject would interpret the first premise as having either one of two distinct meanings: $\mathrm{A}$ and $\mathrm{B}$ are equivalent sets or $\mathrm{A}$ is a proper subset of $B$. (The logical subject is defined as that subject who interprets the premises as they are interpreted in logic textbooks. The question of whether other subjects should also be identified as logical is not of concern at the moment.) The ideal or logical subject would interpret the second premise as having a similar set of two distinct meanings. If the logical subject were asked to indicate whether the conclusion "No C are A" were possibly true, the answer would have to be "yes."

However, suppose a subject does not know that "All
$A$ are B" can be interpreted as A is a subset of B ("subset" will be used in place of "proper subset" throughout the rest of the paper) or that "All C are B" can be interpreted as $C$ is a subset of $B$. Then this subject would conclude that "No C are A" cannot possibly follow from the premises.

A number of different explanations for the misinterpretations subjects give to the premises have been put forward. The various explanations have one thing in common. In particular, they each allow for the possible misinterpretation of "some" as "some but not all" in both the premises "Some A are B" and "Some A are not B." Otherwise, the explanations differ substantially from each other.

Chapman and Chapman (1959) argue that misinterpretation exists because a subject assumes that both a premise and its converse are true. For example, suppose a subject assumes that the premise "All A are B" and its converse, "All B are A," are both true. Then the subject is in effect interpreting the premise "All A are B" as the compound statement "All A are B and all B are A." Note that the logical interpretation of "All A are B" and the Chapman and Chapman interpretation of "All $A$ are B" are not identical (see Table 1, logical and Chapman and Chapman interpretations of "All $A$ are B"). The logical and Chapman and Chapman interpretations of "Some A are not B" also differ (under either interpretation of "some"; i.e., the logical interpretation or the "some-but-not-all" interpretation). Finally, note that the logical and Chapman and Chapman interpretations of "Some A are B" differ when "some" is interpreted as "some but not all."

The motivation for the Chapman and Chapman (1959) model is intuitively compelling. Consider a premise of the form "Some A are not B." Chapman and Chapman argue that subjects will accept the converse of the premise because the converse is of ten true in the real world. For example, the statements "Some plants are not green" and "Some green things are not plants" are both true statements about experience. Chapman and Chapman offer a different explanation of why subjects accept the converse of a premise of the form "All $A$ are B." They suggest that subjects interpret "are" as "are equal to" rather than as "are included in." They give as an example the statements "All right angles are 90 deg" and "All 90-deg angles are right angles." Chapman and Chapman conclude that conversion can explain many of the subjects' errors.

Revlis (1975b) argues for a somewhat stronger version of the conversion hypothesis. He suggests that each subject encodes a premise as its converse only. For example, the premise "All A are B" is encoded as "All $B$ are A." The effect of the type of conversion suggested by Revlis on the final interpretation of the premises is presented in Table 1 (see Revlis model). Revlis concludes that his model can explain much of the observed behavior of subjects in the syllogism task.

Erickson (1978) suggests that subjects choose only 
Table 1

Three Different Models of Premise Interpretation

\section{Logical Model}

A11 $A$ are $B$

$\mathrm{AB}$

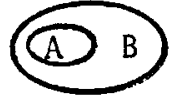

All $A$ are $B$

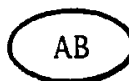

No $A$ are $B$

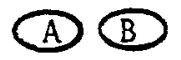

Some A are B

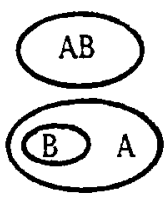

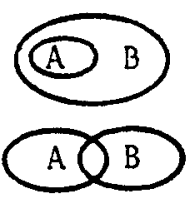

B

Some A are not B

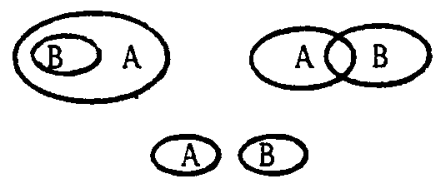

Chapman \& Chapman Mode1

No $A$ are $B$

Some A are B

Some $A$ are not $B$
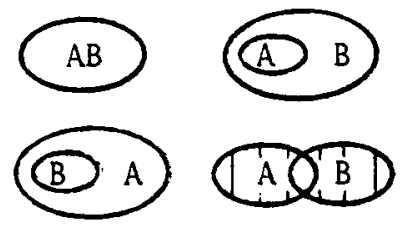

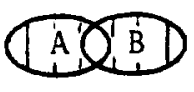

(A) (B)

Revlis Mode 1

A11 A are B

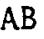

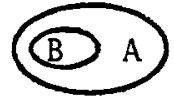

Some A are B

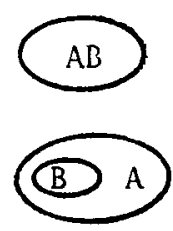

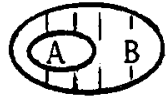
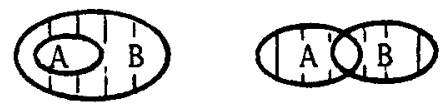

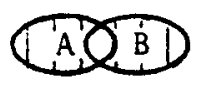

(A) B

Note-Both open and shaded set relations are legitimate interpretations of "Some $A$ are $B$ " and "Some $A$ are not $B$ " if "Some" is interpreted logically. Only the shaded set relations are legitimate interpretations of "Some $A$ are $B$ " and "Some $A$ are not $B$ " if "Some" is interpreted as "Some but not all."

one of the several logical interpretations available for a particular premise (see also Erickson, 1974; Erickson, Wells, \& Traub, Note 3). He determined the preferred interpretation by asking subjects to draw a Venn diagram representation of each of the four premises. (Venn diagrams are circular-shaped representations of a premise; Euler diagrams can assume any shape.) Erickson constructed a model of the syllogistic reasoning process that bases its predictions on the above empirically determined preferences. The relatively good fit of the model leads Erickson to conclude that misinterpretation could explain many of the incorrect responses. Erickson argues that subjects fail to give all logical interpretations because they engage in a less-than-complete analysis of each of the premises. However, Erickson does not go on to suggest why subjects might act on a partial analysis of the premises. Erickson concludes that misinterpretation is widespread.

Guyote and Sternberg (Note 1) suggest a still different model of premise interpretation. The number of interpretations or representations encoded for any one premise is assumed to be a function of four parameters: $e_{1}, e_{2}, e_{3}$, and $e_{4}$, where $e_{1}$ is the probability of encoding only one representation, $e_{2}$ is the probability of encoding exactly two representations, and so on. Furthermore, it was assumed that subjects preferred to encode a premise as its more simple representations (e.g., an equivalence relation) than as its more complex representations (e.g., a subset relation). Guyote and Sternberg's findings do not confirm the general conclusions of Chapman and Chapman (1959), Erickson (1978), or Revlis (1975b). In particular, Guyote and Sternberg 
conclude that misinterpretation of the premises plays an insignificant role in syllogistic reasoning behavior.

Ceraso and Provitera (1971) do not offer a model of premise misinterpretation per se. However, they do offer a test of the role of misinterpretation. Ceraso and Provitera gave subjects two sets of syllogisms. One set consisted of the traditional statement of the premise (e.g., "All A are B"). A second set consisted of a disambiguated or modified statement of the premise (e.g., "All A are B" was restated as "All A are B and all $B$ are A"). It should be noted that Ceraso and Provitera used concrete instantiations of the above abstract syllogisms. For example, subjects saw "No red blocks have holes" instead of "No A are B." Ceraso and Provitera found that subjects made many fewer errors when the premises were modified as indicated. Therefore, they conclude that misinterpretation of the premises significantly affects subjects' behavior.

There are two important sources of disagreement in the above models. First, Guyote and Sternberg (Note 1) conclude that misinterpretation plays an insignificant role, whereas the remaining four studies conclude that misinterpretation plays a significant role. Second, the studies of Chapman and Chapman (1959), Erickson (1978), and Revlis (1975b) each offer a different account of misinterpretation.

A resolution of sorts is possible. Suppose it is assumed that subjects interpret a premise in isolation just as they do in the syllogism task. And suppose subjects are not informed of the meaning of any of the premises. Then Guyote and Sternberg (Note 1) would predict that subjects will interpret all premises correctly (with the possible exception of the misinterpretation of "some" as "some but not all"). Erickson (1978) would predict that one and only one interpretation will be selected for each premise. And Chapman and Chapman (1959) and Revlis (1975b) would make just those predictions listed in Table 1 (and discussed in the above review of their work). In short, a determination of subjects' interpretation of the premises in isolation could potentially rule out one or more of the various competing models.

One might argue that the assumption needed to make the above predictions is itself questionable. That is, one might want to argue that subjects will interpret the premises in isolation differently from the way they do in a standard syllogism task. However, such an argument would seem to run counter to the spirit of at least three of the models (Chapman \& Chapman, 1959; Revlis, 1975b; Guyote \& Sternberg, Note 1) since the models assume that factors quite outside the task determine the actual interpretation given the premises. Erickson (1978), on the other hand, would appear to have a solid objection to the above assumption. If subjects are performing an incomplete analysis of the premises, one might well expect the analysis to be more complete in Experiment 1 than in the standard syllogism task. Therefore, the number of subjects in agreement with the Erickson model may be underestimated by the method used in Experiment 1 (however, see Guyote and Sternberg's analysis of Erickson's model).

\section{EXPERIMENT 1}

\section{Method}

Twenty undergraduates at the University of Michigan participated in the first experiment. Their participation satisfied an introductory psychology course requirement.

Each subject received four premises: "All Doctors are Kuls," "No Doctors are Kuls," "Some Doctors are Kuls," and "Some Doctors are not Kuls." Underneath each premise appeared eight conclusions: "All Doctors are Kuls," "No Doctors are Kuls," "Some Doctors are Kuls," "Some Doctors are not Kuls," "All Kuls are Doctors," "No Kuls are Doctors," "Some Kuls are Doctors," and "Some Kuls are not Doctors." Each subject was asked to indicate whether a conclusion was possibly true or necessarily false. (These two responses were all that were needed to differentiate among the likely interpretations of the four premises. The response "necessarily true" was not needed in the present context.) Subjects were allowed as much time as needed to finish the experiment.

Each subject received a unique ordering of premises and conclusions. The same ordering of conclusions was maintained across all four premises.

The paradigm reported above is similar in some respects to the paradigm used by Sternberg and Turner (Note 2). However, their paradigm has several advantages over the one reported here. Unfortunately, their work did not come to this author's attention until after the present experiment was completed.

\section{Results}

The results of the first experiment are displayed in Table 2. The method used to determine each subject's interpretation of the premises is discussed in detail below. Only 18 subjects were eventually included in the analysis. One subject did not complete the experi-

Table 2

Subjects' Interpretations of a Premise in Experiment 1 as Possible Set Relations

\begin{tabular}{|c|c|c|c|c|}
\hline $\begin{array}{l}\text { Sub- } \\
\text { ject }\end{array}$ & $\begin{array}{l}\text { All A } \\
\text { are B }\end{array}$ & $\begin{array}{l}\text { No A } \\
\text { are B }\end{array}$ & $\begin{array}{c}\text { Some } \mathbf{A} \\
\text { are } \mathrm{B}\end{array}$ & $\begin{array}{c}\text { Some A } \\
\text { are not B }\end{array}$ \\
\hline 1 & $(\mathrm{EQ}, \mathrm{SB})$ & (DJ) & $(\mathrm{EQ}, \mathrm{SB}, \mathrm{SP}, \mathrm{OV})$ & $(\mathrm{SP}, \mathrm{OV}, \mathrm{DJ})$ \\
\hline 2 & (EQ,SB) & (DJ) & (EQ,SB,SP,OV) & $(\mathrm{SP}, \mathrm{OV})$ \\
\hline 3 & (SB) & (DJ) & (OV) & (OV) \\
\hline 4 & (SB) & (DJ) & (OV) & (OV) \\
\hline 5 & $(\mathrm{EQ}, \mathrm{SB})$ & (DJ) & (EQ,SB,SP,OV) & (OV,DJ) \\
\hline 6 & (EQ,SB) & (DJ) & (EQ,SB,SP,OV) & (OV,DJ) \\
\hline 7 & (EQ,SB) & (DJ) & (SP,OV) & (OV,DJ) \\
\hline 8 & (EQ) & (DJ) & (OV) & (OV) \\
\hline 9 & (EQ,SB) & (DJ) & (SP,OV) & (SP,OV) \\
\hline 10 & (SB) & (DJ) & (OV) & (OV) \\
\hline 11 & (EQ) & (DJ) & (OV) & (OV) \\
\hline 12 & $(\mathrm{EQ})$ & (DJ) & $(\mathrm{OV})$ & (SP,OV) \\
\hline 13 & (SB) & (DJ) & (OV) & (OV) \\
\hline 14 & (SB) & (DJ) & (EQ,SB,SP,OV) & (OV) \\
\hline 15 & $(\mathrm{EQ})$ & (DJ) & (EQ,SB,SP,OV) & $(\mathrm{SP}, \mathrm{OV}, \mathrm{DJ})$ \\
\hline 16 & (SB) & (DJ) & (EQ,SB,SP,OV) & (OV) \\
\hline 17 & $(\mathrm{EQ})$ & (DJ) & (OV) & (OV) \\
\hline 18 & $(\mathrm{EQ}, \mathrm{SB})$ & (DJ) & (OV) & (OV) \\
\hline
\end{tabular}

Note $-E Q=A$ and $B$ are equivalent sets. $S B=A$ is a subset of $B . S P=A$ is a superset of $B . O V=A$ and $B$ overlap. $D J=A$ and $B$ are disjoint. 
ment. A second subject was excluded because the subject did not indicate that the conclusion "All A are B" was possibly true when given the premise "All A are B"; the subject did not indicate that the conclusion "No $A$ are $B$ " was possibly true when given the premise "No A are B," and so on.

Only one subject was completely logical (Subject 1 in Table 2). Only two subjects gave all logically possible interpretations to "Some A are not B." And only seven subjects gave all logically possible interpretations to "Some A are B" and "All A are B." Overall, the subjects interpreted $60 \%$ of the 32 conclusions from the four premises in a strictly logical fashion.

The analyses of the Chapman and Chapman (1959), Erickson (1978), and Revlis (1975b) models reported below include only 17 of the 18 subjects (the 1 completely logical subject is excluded because the above three models focus on misinterpretations; the models each allow for the possibility of logical subjects). All 18 subjects are included in the analysis of the Guyote and Sternberg (Note 1) model.

Relatively few subjects gave interpretations of all four premises that were consistent with a given model: Three subjects (Subjects 8, 11, and 17 in Table 2) agreed with Chapman and Chapman (1959), no subjects agreed with Revlis (1975b), seven subjects (Subjects 3, $4,8,10,11,13$, and 17) agreed with Erickson (1978), and three subjects (Subjects 1, 2, and 9) agreed with Guyote and Sternberg (Note 1). (Recall that for all models the interpretation of "some" as "some but not all" was considered acceptable.)

The models perform somewhat better when one considers the proportion of interpretations consistent with a given model across all subjects and across the three premises "All A are B," "Some A are B," and "Some A are not B" ("No A are B" is excluded because each model makes the same predictions for this premise).
The Chapman and Chapman (1959) and Erickson (1978) models do best (64.7\% and 58.8\%, respectively). The Guyote and Sternberg (Note 1) model fares somewhat less well (38.9\%), and the Revlis (1975b) model does poorest of all $(11.8 \%)$.

The models perform still better when one considers the proportion of interpretations consistent with a given model across all subjects within a single premise for certain selected premises. All models correctly predict the interpretations of "No A are B." The Erickson (1978) model agrees with at least half of the interpretations of the three remaining premises $(64.7 \%$ for "All $\mathrm{A}$ are B," $52.9 \%$ for "Some A are B," and $58.8 \%$ for "Some A are not B"). The Chapman and Chapman (1959) model agrees with at least half of the interpretations for two of the remaining premises $(88.2 \%$ for "Some A are B" and 76.4\% for "Some A are not B"). And the Guyote and Sternberg (Note 1) model agrees with at least half of the interpretations for only one of the remaining premises ( $50.0 \%$ for "Some A are not B").

The categorization of a subject's interpretation of a premise as a particular set relation or group of set relations was, in general, straightforward. The categorization rules are summarized in Table 3. For example, the subject who represents "Some A are not B" as an overlap set relation will respond "true" to the conclusions "Some A are B," "Some A are not B," "Some B are A," and "Some B are not A." The subject who represents "Some A are not B" as a superset relation (i.e., as A is a superset of B) will respond "true" to the conclusions "Some A are B," "Some A are not B," "All B are A," and "Some B are A." The subject who represents "Some A are not B" as both an overlap and superset relation will respond "true" to the union of the above conclusions (see Table 3 ).

Of the 72 premises analyzed (4 premises for each of 18 subjects), only 5 could not be interpreted using the

Table 3

Rules Used to Determine the Interpretation of a Premise

\begin{tabular}{|c|c|c|c|c|c|c|c|c|c|}
\hline \multirow[b]{2}{*}{ Premise } & \multicolumn{8}{|c|}{ Conclusion } & \multirow[b]{2}{*}{ Interpretation } \\
\hline & A & $\mathrm{E}$ & I & 0 & $\mathbf{A}^{\prime}$ & $\mathrm{E}^{\prime}$ & $\mathrm{I}^{\prime}$ & $\mathrm{O}^{\prime}$ & \\
\hline All $A$ are $B$ & $\begin{array}{l}\mathrm{T} \\
\mathrm{T} \\
\mathrm{T}\end{array}$ & $\begin{array}{l}F \\
F \\
F\end{array}$ & $\begin{array}{l}\text { T-F } \\
\text { T-F } \\
T-F\end{array}$ & $\begin{array}{l}\mathbf{F} \\
\mathbf{F} \\
\mathbf{F}\end{array}$ & $\begin{array}{l}\mathrm{T} \\
\mathrm{F} \\
\mathrm{T}\end{array}$ & $\begin{array}{l}F \\
F \\
F\end{array}$ & $\begin{array}{l}T-F \\
T-F \\
T-F\end{array}$ & $\begin{array}{l}\mathrm{F} \\
\mathrm{T} \\
\mathrm{T}\end{array}$ & $\begin{array}{l}(\mathrm{EQ}) \\
(\mathrm{SB}) \\
(\mathrm{EQ}, \mathrm{SB})\end{array}$ \\
\hline No $A$ are $B$ & $\mathbf{F}$ & $\mathbf{T}$ & $\mathbf{F}$ & $\mathrm{T}-\mathrm{F}$ & $\mathbf{F}$ & $\mathrm{T}$ & $F$ & $\mathrm{~T}-\mathrm{F}$ & (DJ) \\
\hline Some $A$ are $B$ & $\begin{array}{l}F \\
F \\
T\end{array}$ & $\begin{array}{l}F \\
F \\
F\end{array}$ & $\begin{array}{l}\mathrm{T} \\
\mathrm{T} \\
\mathrm{T}\end{array}$ & $\begin{array}{l}\mathrm{T} \\
\mathrm{T} \\
\mathrm{T}\end{array}$ & $\begin{array}{l}\mathrm{F} \\
\mathrm{T} \\
\mathrm{T}\end{array}$ & $\begin{array}{l}F \\
F \\
F\end{array}$ & $\begin{array}{l}\mathrm{T} \\
\mathrm{T} \\
\mathrm{T}\end{array}$ & $\begin{array}{l}\mathrm{T} \\
\mathrm{T} \\
\mathrm{T}\end{array}$ & $\begin{array}{l}\text { (OV) } \\
\text { (SP,OV) } \\
(\mathrm{EQ}, \mathrm{SB}, \mathrm{SP}, \mathrm{OV})\end{array}$ \\
\hline Some $A$ are not $B$ & $\begin{array}{l}F \\
F \\
F \\
F\end{array}$ & $\begin{array}{l}\mathrm{F} \\
\mathrm{F} \\
\mathrm{T} \\
\mathrm{T}\end{array}$ & $\begin{array}{l}\mathrm{T} \\
\mathrm{T} \\
\mathrm{T} \\
\mathrm{T}\end{array}$ & $\begin{array}{l}\mathrm{T} \\
\mathrm{T} \\
\mathrm{T} \\
\mathrm{T}\end{array}$ & $\begin{array}{l}\mathrm{F} \\
\mathrm{T} \\
\mathrm{F} \\
\mathrm{T}\end{array}$ & $\begin{array}{l}\mathbf{F} \\
\mathbf{F} \\
\mathbf{T} \\
\mathbf{T}\end{array}$ & $\begin{array}{l}\mathrm{T} \\
\mathrm{T} \\
\mathrm{T} \\
\mathrm{T}\end{array}$ & $\begin{array}{l}\mathrm{T} \\
\mathrm{T} \\
\mathrm{T} \\
\mathrm{T}\end{array}$ & $\begin{array}{l}\text { (OV) } \\
\text { (SP,OV) } \\
\text { (OV,DJ) } \\
\text { (SP,OV,DJ) }\end{array}$ \\
\hline
\end{tabular}

Note $-A=$ all $A$ are $B ; E=$ no $A$ are $B ; I=$ some $A$ are $b ; O=$ some $A$ are not $B ; A^{\prime}=$ all $B$ are $A ; E^{\prime}=$ no $B$ are $A ; I^{\prime}=$ some $B$ are $A$; $O^{\prime}=$ some $B$ are not $A . ' E Q=A$ and $B$ are equivalent sets; $S B=A$ is a subset of $B ; S P=A$ is a superset of $B ; O V=A$ and $B$ overlap; $D J=A$ and $B$ are disjoint. The subject can mark " $T-F$ " conclusions as possibly true or necessarily false without affecting the interpretation of the premise. Only those rules needed to determine interpretations actually used by subjects in Experiment 1 are categorized. 
above rules. In each of the five cases, it was not clear which one of two interpretations should hold. The more logically complete interpretation was chosen in each of the five cases.

\section{Discussion}

No one model does an especially good job of explaining the overall behavior of subjects. However, the Chapman and Chapman (1959) model predicts almost all of the interpretations of two of the four premises ("No A are B" and "Some A are B") and the majority of the interpretations of a third premise ("Some A are not B"). The fact that the Chapman and Chapman model predicts so few of the interpretations of "All A are B" does not imply that the entire model needs reformulating, since the explanation for the misinterpretation of "All A are B" is different from the explanation for the misinterpretations of the other premises. Unfortunately, the present experiment does not suggest what the correct explanation for the misinterpretation of "All A are B" might be.

All of the various models of misinterpretation predict at least two possible interpretations for one or more premises. The models do not indicate which interpretation a subject will actually choose. It is quite clear from Table 2 that subjects do differ greatly in their choice of interpretations. This implies that in order to study the precise effect of premise misinterpretation on syllogistic reasoning behavior, it will be necessary to construct models of syllogistic reasoning that base their predictions on the interpretations specific to each subject. Such models are developed in the next section. Note that while the models do not assume that different subjects have the same interpretation of a given premise, the models do assume that a particular subject will have one and only one interpretation of any given premise (at least throughout the course of the experiment).

\section{MODELS OF PREMISE INTERPRETATION}

Four models of syllogistic reasoning are constructed in this section: the logical model, the premise model, the combination model, and the readout model. Each model assumes that the processing of a syllogism is a five-stage process: encoding, selection, combination, readout, and response. These general stages are by no means unique to this paper (Erickson, 1974; Revlis, 1975 b; Sternberg \& Turner, Note 2). There may be one or more steps or operations in each of the five stages. The models predict a subject's responses to any given syllogism.

As one might well expect, the logical model assumes that all five stages are logical. (The distinction between logical and alogical in this section is purely one of convenience. A stage is defined as logical if the input and output of that stage are related in a fashion that, loosely speaking, can be said to agree with formal logic.
Otherwise, the stage is defined as alogical.) The logical model is noted in the text as (P-Log, C-Log, R-Log). "P-Log"stands for a logical premise encoding operation, "C-Log" stands for a logical combination operation, and "R-Log" stands for a logical readout operation. Since all models assume logical selection and response stages, there was no need for additional components associated with these stages. The premise model is noted as (P-Alog, C-Log, R-Log). The first operation, the encoding of the premise (P-Alog), is alogical. The combination model is noted as (P-Alog, C-Alog, R-Log), and the readout model is noted as (P-Alog, C-Alog, R-Alog). Each model incorporates successive alogical components. The reader will note that four of the eight $\left(2^{3}\right)$ models one might test with binary premise, combination, and readout components are not examined in this paper. These four models prove to be of little interest (Fisher, Note 4).

In order to provide a test of the predictions of the various models, subjects in Experiment 1 were run in a second experiment. They were asked to indicate whether each of four conclusions to a particular syllogism was possibly true or necessarily false. Note that syllogism studies have typically included the responses "necessarily true" and "none of the above." However, subjects have a bias against accepting the response "none of the above" (Chapman \& Chapman, 1959; Dickstein, 1975; Revlis, 1975b; but see also Dickstein, 1976). This bias can obscure the exact misinterpretation of the premises that originally governed subjects' behavior. Using "necessarily true" as a response category (Guyote \& Stemberg, Note 1) can also obscure the role of premise interpretation, since the determination that a conclusion is necessarily true may well depend on a prior determination of which conclusions are possibly true.

\section{Logical Model}

The logical model (P-Log, C-Log, R-Log) assumes that the premises of the syllogisms are interpreted correctly. For example, suppose the subject is given a syllogism whose major premise is "Some B are not A" and whose minor premise is "No C are B." Then the logical subject would interpret "Some B are not A" as one of three set relations between $B$ and $A$ : (1) $B$ is a superset of A, (2) B and A overlap, or (3) B and A are disjoint (see Table 4, encoding stage). The logical subject would interpret "No C are B" as a disjoint set relation between $\mathrm{C}$ and $\mathrm{B}$. The selection stage follows the encoding or premise interpretation stage. In this stage, the subject must select or identify all pairs of set relations to be analyzed. A pair of set relations consists of a set relation between $B$ and $A$ from the first premise and a set relation between $C$ and $B$ from the second premise. In this case, the subject must select three pairs of set relations for further analysis: (1) $B$ is a superset of $A$ and $C$ and $B$ are disjoint, (2) B and $A$ overlap and $\mathrm{C}$ and $\mathrm{B}$ are disjoint, and (3) $\mathrm{B}$ and $\mathrm{A}$ are disjoint and $\mathrm{C}$ and $B$ are disjoint (see Table 4 , selection stage). An 
Table 4

The Logical Model (P-Log, C-Log, R-Log)

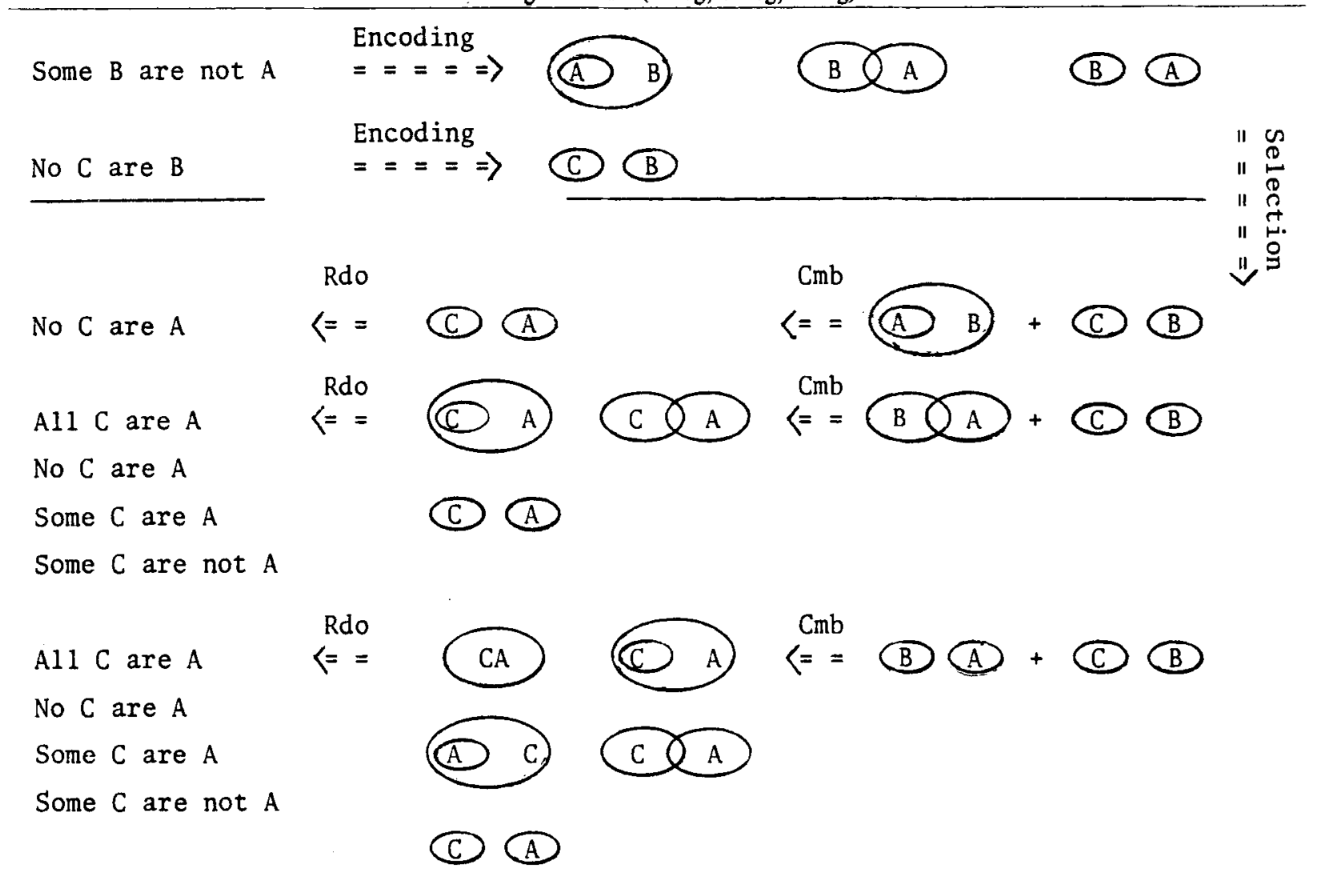

Note - Rdo $=$ the readout stage $; C m b=$ the combination stage .

analysis of each pair will yield a series of derived or combined set relations between $\mathrm{C}$ and $\mathrm{A}$ (see Table 4, combination stage). Finally, in the readout stage, the subject must map each derived set relation between $C$ and $\mathrm{A}$ into one or more conclusions (see Table 4 , readout stage). For example, the derived set relation, $\mathrm{C}$ is a subset of $A$, gets mapped into the possibly true conclusions "All C are A" and "Some C are A."

A distinction was referred to above that should be given more pointed emphasis. The terms "derived set relations" and "combined set relations" will always refer to one or more of the five set relations: $C$ and $A$ are equivalent sets, $C$ is a subset of $A, C$ is a superset of $\mathrm{A}, \mathrm{C}$ and $\mathrm{A}$ overlap, and $\mathrm{C}$ and $\mathrm{A}$ are disjoint. The term "conclusions" will always refer to one or more of the four conclusions: "All C are A," "No C are A," "Some C are A," and "Some C are not A." It should also be noted that a derived or combined set relation will frequently be referred to simply as a combination.

$\dot{A}$ computer program that generated the response ("possibly true" or "necessarily false") to each of the four conclusions to the 64 different syllogisms was written. While it is easy enough to generate the responses to the logical model by hand, this is not true of the other models tested. The FORTRAN code used for the various models is available upon request from the author.

\section{Premise Model}

The premise model (P-Alog, C-Log, R-Log) allows for the misinterpretation of the premises of a syllogism. The model bases its predictions for a subject on the subject's interpretation of the premises. Subjects with different interpretations will, in general, arrive at different conclusions. The steps of the premise model are outlined in Table 5. Suppose a subject interprets the premises in Experiment 1 as indicated in Table 5 . This subject is assumed to give the same interpretation to the premises when they appear in a syllogism in Experiment 2. Therefore, the subject is assumed to interpret the first premise, "Some B are not A," as an overlap set relation. Note that this qualifies as a misinterpretation (see the logical interpretation of "Some B are not A," above). The subject interprets "No C are B" correctly. The remaining steps of the premise model are the same as the logical model and need not be worked through.

\section{Combination Model}

The combination model (P-Alog, C-Alog, R-Log), like the premise model, bases its predictions for a subject in Experiment 2 on that subject's interpretation of the premises in Experiment 1. However, unlike the premise model, the combination model assumes that the subject will not form all the derived or combined set relations 
Table 5

Experiment 1: premise misinterpretation
Al1 A are B
$\mathrm{AB}$
No $A$ are $B$
(A) (B)
Some A are B

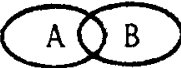
Some A are not B

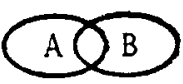

Experiment 2: predictions of premise model based on above misinterpretations
Some $B$ are not $A$
Encoding
No $C$ are $B$
Encoding
$===\Rightarrow$

All C are $A$
Readout
$\Leftarrow==$

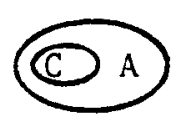
Some $\mathrm{C}$ are $\mathrm{A}$
Some $\mathrm{C}$ are $\mathrm{A}$
Readout

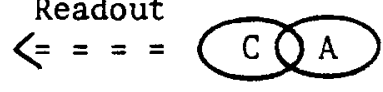
Some $\mathrm{C}$ are not $\mathrm{A}$
No $\mathrm{C}$ are $\mathrm{A}$
$\stackrel{\text { Readout }}{==}=$ (C)
Some $\mathrm{C}$ are not $\mathrm{A}$

B $A$

Comb.

$E==B A+C B$

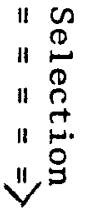

between the subject and predicate of the syllogism. Specifically, it is assumed that the subject will form only those derived set relations that the subject gives evidence of understanding in Experiment 1 . An example is presented in Table 6. Note that the subject never interprets a premise as a subset or superset relation in Experiment 1. Therefore, the subject may not derive the subset or superset relations in the combination stage, even though such relations are a logical consequence of the derivation. The examples in Tables 5 and 6 show that the premise and combination models can lead to different predictions. The combination in
Table 5 (Experiment 2) of an overlap set relation between $B$ and $A$ and a disjoint set relation between $C$ and $B$ leads to three derived set relations: (1) $C$ is a subset of A, (2) C and A overlap, and (3) C and A are disjoint. The first subset relation is not rejected in the premise model. However, this derived set relation is rejected in the combination model. Thus, note that in Table 6 (Experiment 2) only two set relations are derived in the combination stage: (1) $\mathrm{C}$ and $\mathrm{A}$ overlap and (2) $\mathrm{C}$ and $\mathrm{A}$ are disjoint. In short, the combination model predicts that the subject will indicate only three conclusions are possibly true-"No C are A," "Some C 
Table 6

Experiment 1: premise misinterpretation

All A are B

No $A$ are $B$

Some A are B

Some $A$ are not $B$

\section{$A B$}

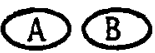

Experiment 2: predictions of combination model based on above misinterpretations

Some $B$ are not $A$

No $C$ are $B$

Some $\mathrm{C}$ are $\mathrm{A}$

\section{Encoding}

$$
==\stackrel{=}{=}
$$

Encoding

$===\Rightarrow$
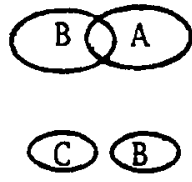

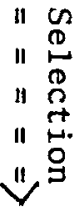

Some $C$ are not $A$

No $C$ are $A$

Readout

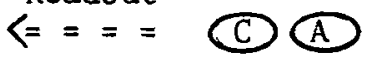

Some $\mathrm{C}$ are not $\mathrm{A}$

are $\mathrm{A}$," and "Some $\mathrm{C}$ are no $\mathrm{A}$ "-whereas the premise model predicts that all conclusions are possibly true.

\section{Readout Model}

The readout model (P-Alog, C-Alog, R-Alog) incorporates the alogical components of the above two models and adds an additional alogical readout component. In the combination model, it was assumed that every derived set relation that could be constructed (as indicated by Experiment 1) was translated into all of the associated logical conclusions. This assumption is not made in the readout model. A derived set relation is translated into a conclusion in the readout model (Experiment 2) only if a subject maps a conclusion into the derived set relation in Experiment 1 (the mapping from conclusions into derived set relations can be inferred from the mapping of premises into set relations; e.g., see Table 7, Experiment 1). This can mean that some derived set relations map into no conclusions whereas other derived set relations map into fewer than the complete set of logical conclusions. An example is presented in Table 7. The combination stage (Experiment 2) leads to three derived set relations: (1) $\mathrm{C}$ is a subset of $A,(2) C$ and $A$ overlap, and (3) C and $A$ are disjoint. Note that no conclusion in Experiment 1 maps into the derived set relation $\mathrm{C}$ is a subset of $\mathrm{A}$. Therefore, this derived set relation is not mapped into a conclusion in Experiment 2. Further note that in Experiment 1 "Some $\mathrm{C}$ are not $\mathrm{A}$ " is the only conclusion that maps into the derived set relation $\mathrm{C}$ and $\mathrm{A}$ overlap. Therefore, the derived overlap set relation is mapped into only one conclusion, "Some $\mathrm{C}$ are not A."

Finally, note that the conclusion "Some $\mathrm{C}$ are not $A$ " appears in parentheses below the conlusion "No C are A." Given the conclusion "No C are A," some subjects may decide that "Some $\mathrm{C}$ are not $\mathrm{A}$ " logically 
Table 7

Experiment 1: premise and conclusion misinterpretation

All A are B

No $A$ are $B$

Some A are B

Some A are not B

Al1 C are A

No $C$ are $A$

Some $\mathrm{C}$ are $\mathrm{A}$

Some $C$ are not $A$

\section{$\mathrm{AB}$}

(A) (B)
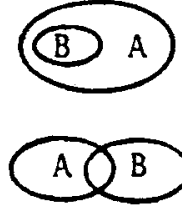

$\mathrm{CA}$

Experiment 2: predictions of the readout mode1 based on above misinterpretations

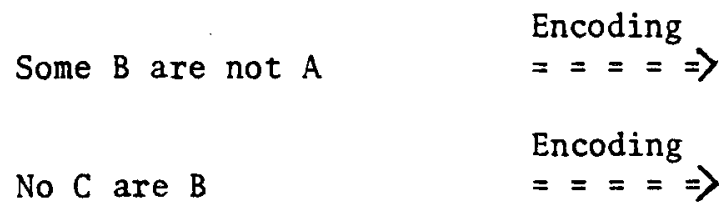

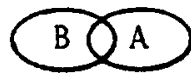

(C) (B)

$\| \stackrel{0}{\pi}$

I $\stackrel{+}{+}$

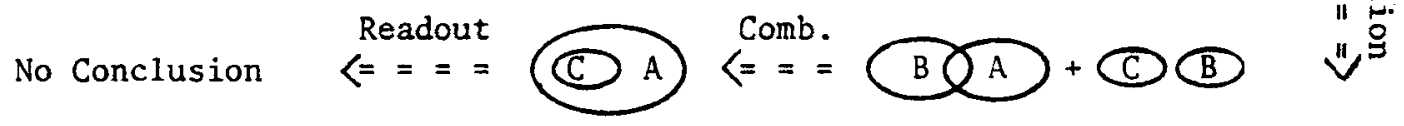

Readout

Some $C$ are not $A \Leftrightarrow===$

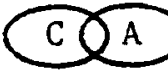

Readout

No $C$ are $A$

$\Leftarrow===$ C $\mathrm{A}$

(Some $\mathrm{C}$ are not A)

follows. Other subjects may decide that "Some $\mathrm{C}$ are not A" does not follow. The premise interpretation data from Experiment 1 can be and was used to determine which decision a subject will make in Experiment 2. Similarly, given the conclusion "All C are A," some subjects may decide that "Some $\mathrm{C}$ are $\mathrm{A}$ " logically follows, whereas other subjects may decide that "Some $\mathrm{C}$ are $\mathrm{A}$ " does not logically follow. Again, the evidence from Experiment 1 was used to decide which choice a subject would make in Experiment 2. This completes the discussion of the various models that allow for premise misinterpretation. 


\section{EXPERIMENT 2}

\section{Method}

The 20 subjects who participated in Experiment 2 also had participated in Experiment 1. The second experiment followed immediately after the first. Each subject saw 64 syllogisms (four distinct figures for each of four major and four minor premises). "Kuls" was used as the predicate of the major premise, "Sats" was used as the subject of the minor premise, and "Doctors" served as the middle term. Each syllogism was followed by four conclusions: "All Sats are Kuls," "No Sats are Kuls," "Some Sats are Kuls," and "Some Sats are not Kuls." Subjects were asked to indicate whether a conclusion was possibly true or necessarily false. Subjects were allowed as much time to finish the experiment as they needed.

Each subject saw a different ordering of the 64 syllogisms. The ordering of conclusions in the first and second experiments remained identical for any one subject but varied between subjects.

The choice of nonsense words for subject and predicate and a meaningful word for the middle term was made for two reasons. On the one hand, the use of meaningful words for all three terms might bias the subject in undesirable ways. For example, if "Lawyers" were the subject and "Cooks" the predicate, a factual bias to the conclusion "All Lawyers are Cooks" might well exist. Such biases, while important, were not the focus of this study. On the other hand, the use of meaningless words for all three terms might lead subjects to use strategies altogether different from those used in a more natural setting. It was hoped that the present choice of words would provide some sort of reasonable middle ground.

\section{Results}

The responses of each subject were recorded as a zero or a one. A one was entered if the subject indicated that a conclusion was possibly true; a zero was entered otherwise. The recorded responses were then summed across all 18 subjects within each of the 256 conclusions. The predictions of a model were also recorded as a zero or a one and summed across all 18 subjects within each of the 256 conclusions. The percentage of the variance of the observed responses explained by the predicted responses was chosen as a measure of fit. Other measures of fit were examined by Fisher (Note 4) and were found to be in good agreement with the explained variance.

The logical model fared rather poorly by comparison with other models. It explained only $30 \%$ of the variance. The premise model explained $48 \%$ of the variance, the combination model explained $51 \%$ of the variance, and the readout model explained $49 \%$ of the variance.

The proportion of responses that agreed with the predictions of the premise model differed significantly from the proportion of responses that agreed with the predictions of the logical model [McNemar's test of correlated proportions: $\left.\chi^{2}(1)=91.2, p<.001\right]$. The proportion of responses in agreement with the combination model differed significantly from the proportion of responses in agreement with the premise model $\left[x^{2}(1)=22.04, p<.001\right]$. The readout model did not perform significantly better than either the premise model or the combination model.

\section{Discussion}

There are two major differences between the models of misinterpretation developed in this paper and earlier models of misinterpretation.

First, the current models can be used to determine the influence of the complete range of possible misinterpretations, whereas earlier models were tied to a specific set of misinterpretations. As a consequence, the present models enjoy a measure of generality beyond that of earlier models.

Second, the current models assume that premise misinterpretation can have important effects throughout the syllogistic reasoning process, effects that extend beyond the encoding stage and into the combination and readout stages. This broad view of premise misinterpretation seems to introduce a reasonable portion of consistency into a subject's behavior. It was therefore somewhat surprising to find that the combination and readout models fared only slightly better than the premise model. However, inspection of the results from Experiment 1 (Table 2) indicates that only three subjects would be expected to produce different responses for the premise and combination models (those three subjects who gave no evidence of understanding all the possible set relations). And for these three subjects, one would expect differences on only 21 of 768 different predictions. Note that if a different pattern of misinterpretations had been obtained, the disagreements between the models could have been much more substantial.

It should be emphasized that no parameters are being fit in any of the above models. Thus, it is by no means necessarily the case that the three models of misinterpretation will perform better than the logical model. Specifically, the ith subject's interpretation of the premises (from Experiment 1) is used to predict his of her response to the jth conclusion ("possibly true" or "necessarily false") of the kth syllogism in Experiment 2. Thus, the model can be written as $Y_{i j k}=X_{i j k}$ $+\epsilon$, where $Y_{\mathrm{ijk}}$ is the subject's actual response in Experiment 2 (coded as a one or a zero) and $X_{i j k}$ is the subject's predicted response in Experiment 2 (also coded as a one or a zero). Note that, since the same subjects were used in Experiments 1 and 2, the ith subject is the same in both experiments.

The models that allow for premise misinterpretation clearly perform better than the logical model. The combination model performs best of all. However, because the increase in the performance of the combination model is based on the results from only three subjects and because the difference between the combination and premise models was so slight, only the theoretically more simple of the two models (i.e., the premise model) was chosen for additional study and modification. (Recall that the readout model did not perform significantly better than the premise model and that the readout model was theoretically the most complex of the three models.) 


\section{INFORMATION PROCESSING CAPACITY}

The premise, combination, and readout models explain more variance than the logical model. However, much of the variance is still left unexplained. Recent work by Guyote and Sternberg (Note 1) and Sternberg and Turner (Note 2) suggests a systematic source for the unexplained variance. Specifically, they argue that the limitations of working memory affect behavior at the selection stage. For example, consider the syllogism with major premise "Some A are B" and minor premise "Some $\mathrm{C}$ are B." Both the major and the minor premise can be interpreted as one of four set relations (see Table 1). Therefore, a total of 16 different set relation pairs must be selected for analysis. A subject may well lose track of those pairs of set relations that have and have not been selected. As a consequence, fewer than the complete set of pairs may be selected.

Sternberg and Turner (Note 2) assume that four set relation pairs at most are ever selected. One set relation pair is selected with some probability, say $p_{1}$, two set relation pairs are selected with some (not necessarily) different probability, say $p_{2}$, and so on through four pairs of set relations. Sternberg and Turner also assume that certain set relation pairs are preferred over others. The most preferred single set relation pair consists of a Type 1 set relation from the first and second premise. (A Type 1 set relation is an equivalence set relation, Type 2 set relations are overlap and disjoint set relations, and Type 3 set relations are subset and superset relations.) In general, all pairings of Type 1 with Type 1 and Type 2 are selected first. All pairings of Type 2 with Type 2 representations are selected next. Finally, all other pairings are selected. This ordering is assumed to reflect the ease with which relations of each type are stored and manipulated in memory. The complete details of the model can be found in Sternberg and Turner. They conclude that information processing limitations have a significant effect on the behavior of subjects.

The Sternberg and Turner (Note 2) model will be designated the limited-capacity model (P-Alog, L-Alog, C-Log, R-Log). Note that the limited-capacity component, "L-Alog," is placed between the premise and combination components, since the limited-capacity component refers to aspects of the selection stage. Also note that the limited-capacity model contains an alogical premise interpretation component as well as an alogical selection component. Parameter estimates of $p_{1}, p_{2}, p_{3}$, and $p_{4}$ were taken from Sternberg and Turner. (Additional analyses showed that the use of parameter estimates based on the data from Experiment 2 made very little difference in the amount of variance explained by the limited-capacity model.)

The limited-capacity model proved to be a considerable improvement over the premise model. It will be recalled that the premise model explained $48 \%$ of the variance. The limited-capacity model explains $60 \%$ of the variance. Note that a model (P-Log, L-Alog, C-Log, R-Log) with a limited-capacity component and no premise misinterpretation component explains only $47 \%$ of the variance. This indicates that the influence of the limited-capacity component is not somehow attenuated by the presence of the premise component in the limited-capacity model.

It should be emphasized that none of the parameters for the limited-capacity model were free to vary. Thus, the limited-capacity model could well have explained less variance than the premise model. Recall that the probabilities needed in the limited-capacity model were taken from the Sternberg and Turner (Note 2) study. Formally, let $X_{i j k m}$ be the probability that the ith subject in Experiment 2 will respond "possibly true" to the jth conclusion of the kth syllogism when the ith subject's interpretations of the premises are used (Experiment 1) and when $\mathrm{m}$ set relation pairs are examined. If $\mathrm{p}_{\mathrm{m}}$ is the probability that $\mathrm{m}$ set relation pairs are analyzed, then one obtains: $Y_{\mathrm{ijk}}=\mathrm{p}_{1} \mathrm{X}_{\mathrm{ijk} 1}+$ $\mathrm{p}_{2} \mathrm{X}_{\mathrm{ijk} 2}+\mathrm{p}_{3} \mathrm{X}_{\mathrm{ijk} 3}+\mathrm{p}_{4} \mathrm{X}_{\mathrm{ijk} 4}+\epsilon$. As in the premise model, $Y_{\mathrm{ijk}}$ is the observed response in Experiment 2.

While the limited-capacity model explains fully $60 \%$ of the variance, this still leaves a great deal of the variance unaccounted for. An examination of the evidence from the second experiment suggests the sort of component that may be needed in addition to the premise and limited-capacity components. Consider the syllogism whose first premise is "No B are A" and whose second premise is "No $C$ are B." The premise model predicts that all 18 subjects in Experiment 2 should have indicated that each of the four conclusions to the syllogism is possibly true. For this particular syllogism, the limited-capacity model makes the very same predictions (the demonstration is left as an exercise for the reader). However, nine subjects indicated that "All C are A" was necessarily false, five subjects indicated that "No $\mathrm{C}$ are $\mathrm{A}$ " and "Some C are A" were both necessarily false, and four subjects indicated that "Some C are not A" was necessarily false. Such failures of prediction persist throughout all four figures of the example syllogism. Similar sorts of failures appear in other syllogisms.

In short, the subjects are making a class of mistakes that cannot be explained by either of the models introduced so far. The source of the errors appears to be part of the actual combination or deductive stage. The subjects are not marking all possible conclusions. Therefore, the subjects may not be deriving all the possible set relations between $C$ and $A$ when analyzing a pair of set relations from the first and second premises. Ceraso and Provitera (1971), Erickson (1978), and JohnsonLaird and Steedman (1978) have focused on problems of the combination or deductive stage. Their research is reviewed in the next section. (Note that the problems with the combination stage referred to by the above researchers are altogether different from the problems of the combination stage referred to in earlier discussions of the combination model.) 


\section{DEDUCTIVE STRATEGIES}

Ceraso and Provitera (1971) were among the first to note that subjects might not form all derived set relations or combinations consistent with a single pair of set relations from the first and second premise (it will be recalled that Ceraso and Provitera stated each premise in such a fashion that it could be interpreted as one and only one set relation). They found that subjects made very few errors when only one derived set relation or combination between the subject and predicate of the syllogism could be formed (e.g., $\mathrm{C}$ and $\mathrm{A}$ are equivalent sets is the only set relation that can be derived when $\mathrm{A}$ and $\mathrm{B}$ are equivalent sets and $\mathrm{B}$ and $\mathrm{C}$ are equivalent sets). However, subjects made many more errors when multiple derived set relations between the subject and predicate of the syllogism could be formed (e.g., any set relation between $C$ and $A$ can be derived when $A$ is a subset of $B$ and $C$ is a subset of $B$ ).

Ceraso and Provitera (1971) argue that subjects will frequently label a derived set relation as necessarily false (i.e., the set relation cannot be derived) that, logically, is possibly true (i.e., the set relation can be derived). Such subjects can be described as having incomplete deductive strategies. Ceraso and Provitera rule out the possibility that subjects are using an alogical deductive strategy. They mean by an alogical deductive strategy a strategy that leads a subject to label as possibly true a derived set relation that, logically, is necessarily false.

Erickson et al. (Note 3) had subjects draw the Vennderived set relation that follows necessarily from a pair of Venn premises. Like Ceraso and Provitera (1971), Erickson et al. found that subjects did not always form the correct set relation. However, unlike Ceraso and Provitera, Erickson et al. found that subjects sometimes identified as necessarily true derived set relations that, logically, were necessarily false. Erickson (1978) used these results to predict performance in a conventional syllogism task. He concludes that variations in the deductive strategies contribute significantly to the production of incorrect responses.

Johnson-Laird and Steedman (1978) construct what they define as an analogical theory of reasoning with quantifiers. They abandon the notion that the premises are represented as Venn diagrams. Instead, they argue that a class or set is represented as an arbitrary number of exemplars. This eventually leads Johnson-Laird and Steedman to the prediction that subjects make errors on a syllogistic reasoning task because, among other things, they employ an incomplete deductive strategy. To test this general prediction, Johnson-Laird and Steedman gave subjects syllogisms with concrete premises (e.g., "All of the gourmets are storekeepers; all of the storekeepers are bowlers.") Space was provided for subjects to write down those conclusions that followed necessarily from the premises. Johnson-Laird and Steedman conclude that the results support the notion of an incomplete deductive strategy such as they propose. They cite, for example, the fact that $80.4 \%$ of the responses to syllogisms not affected by an incomplete deductive strategy were correct, whereas only $46.5 \%$ of the responses to syllogisms that could be affected by an incomplete deductive strategy were correct

Taken as a group, the above studies indicate that subjects will on occasion form other than the correct set of combinations from a given pair of set relations. Some true combinations will be labeled as false if subjects are using an incomplete deductive strategy and some false combinations will be labeled as true if subjects are using an alogical deductive strategy. The above studies also indicate that alogical and incomplete deductive strategies may influence behavior in the standard syllogism task. However, one cannot conclude from the above studies that variation in the deductive strategies will be a significant factor in a standard syllogism task after the effects of both misinterpretation and information handling capacity have been partialed out, since none of the above studies controlled for both of these effects.

In order to determine the relative influence of alogical and incomplete deductive strategies, one must construct a model that includes premise misinterpretation and limited-capacity components as well as a deductive component. Accordingly, such a model was constructed and is noted in the paper as the deductive model (P-Alog, L-Alog, D-Alog, R-Log). The deductive component, "D-Alog," is placed between the limitedcapacity component (the selection stage) and the readout component (the readout stage), since the deductive component refers to aspects of the combination stage (recall that the combination stage follows the selection stage and precedes the readout stage). The deductive model assumes that there is some probability (the same probability for all subjects) that a subject will indicate, for any given pair(s) of set relations from the first and second premise, that a specified subset of combinations (e.g., $C$ is a subset of $A, C$ and $A$ are disjoint) is possibly true or necessarily false.

Information on the first two components of the deductive model is currently available (i.e., the inputs and outputs of the premise and selection stages have been determined or are assumed to be known). However, the outputs of the deductive component have not yet been determined. In order to identify these outputs, the deductive component can be studied in isolation. This is done in the next experiment (Experiment 3 ). Specifically, subjects are given the theoretical inputs to the deductive component and asked to respond to various outputs. The experiment is described in detail below. Once the outputs of the deductive component are known, the component (isolable stage) can be introduced as "D-Alog" into the full deductive model (P-Alog, L-Alog, D-Alog, R-Log). One can then determine whether the deductive model fits the data from the standard syllogism task (Experiment 2) better than 
either the limited-capacity model or the premise model. If an improved fit is observed, this will indicate that behavior on the isolated task or component (Experiment 3) may explain aspects of behavior on the more complex task (Experiment 2).

\section{EXPERIMENT 3}

\section{Method}

Twenty-one undergraduates at the University of Michigan participated in the experiment. Their participation satisfied an introductory psychology course requirement.

Subjects were presented with 10 problems. Each problem consisted of two disambiguated premises followed by five set relations or combinations. Subjects were asked to indicate whether each combination was logically possible or necessarily false. An example problem and combinations are presented in Table 8. Subjects were allowed as much time as they needed to finish the experiment.

It should be noted that the modified or disambiguated premise "No block with a green stripe has a red stripe; no block with a red stripe has a green stripe" is redundant. The first statement implies the second. However, some subjects may not be aware of the implication. Therefore, in order to remove all possible ambiguity, the premise was stated redundantly,

Each premise describes one of the five possible set relations that can exist between the terms of the premise. The first premise in Table 8 can therefore be more simply stated as "Green subset Red." The second premise can be more simply stated as "Red overlap Yellow." The problems presented to subjects can now be compactly listed (first premise followed by second premise): (1) "Green equivalence Red, Red subset Yellow," (2) "Green subset Red, Red subset Yellow," (3) "Green subset Red, Red superset Yellow," (4) "Green subset Red, Red overlap Yellow," (5) "Green superset Red, Red subset Yellow," (6) "Green superset Red, Red disjoint Yellow," (7) "Green overlap Red, Red superset Yellow," (8) "Green overlap Red, Red overlap Yellow," (9) "Green overlap Red, Red disjoint Yellow," and (10) "Green disjoint Red, Red disjoint Yellow."

Subjects were given the above 10 problems in a random order. Each subject saw a unique ordering of the combinations. The ordering of the combinations remained the same for any one subject throughout the experiment.

\section{Results}

Several summary descriptions of the data were computed. Sixty-four percent of the responses were logically correct. Twenty-seven percent of the responses (75\% of the errors) were "misses" (i.e., responses marked as necessarily false that, logically, were possibly true). The remaining $9 \%$ of the responses (25\% of the errors) were "false alarms" (i.e., responses marked as possibly true that, logically, were necessarily false). The correlation between the number of subjects one would expect to make a particular response if all subjects were logical with the number of subjects actually making the response was moderately large $(r=.76)$. Finally, the above correlation was computed for each subject. The correlations ranged from .88 to .00 .

A more detailed tabulation of the results is presented in Table 9. Subjects derived the correct set relations $48 \%$ of the time when all five set relations were logically possible (see "SB-SP," "OV-OV," and "DJ-DJ" in Table 9), 67\% of the time when four set relations were logically possible ("SP-SB"), 62\% of the time when three set relations were logically possible ("SB-OV," "SP-DJ," "OV-SP," and "OV-DJ"), and 76\% of the time when only one set relation was logically possible ("EQ. SB" and "SB-SB"). (Note that there is no case in Table 9 in which two and only two set relations were logically possible.)

The results from Experiment 3 were used to construct the deductive component of the deductive model. The deductive model so defined explained fully $74 \%$ of the variance in Experiment 2. The construction of the deductive component proceeded as follows.

It will be recalled that only 10 of the possible 25 pairs of set relations were selected for study. Information on five additional pairs of set relations can be determined indirectly from the above results. Note that Dickstein (1975) finds that the order of the first and second premise is not important in a standard

Table 8

Practice Problem and Conclusions for Experiment 3

Problem

Every block with a green stripe has a red stripe At least one block has a red stripe without a green stripe

At least one block has a red stripe without a yellow stripe At least one block has a yellow stripe without a red stripe At least one block has both a red stripe and a yellow stripe

$$
\text { Conclusions }
$$

Every block with a green stripe has a yellow stripe Every block with a yellow stripe has a green stripe Every block with a green stripe has a yellow stripe At least one block has a yellow stripe without a green stripe Every block with a yellow stripe has a green stripe At least one block has a green stripe without a yellow stripe At least one block has a green stripe without a yellow stripe At least one block has a yellow stripe without a green stripe At least one block has both a green stripe and a yellow stripe No block with a green stripe has a yellow stripe No block with a yellow stripe has a green stripe
FIRST

PREMISE

SECOND

PREMISE

FIRST

COMBINATION

SECOND

COMBINATION

THIRD

COMBINATION

FOURTH

COMBINATION

FIFTH

COMBINATION 
Table 9

Logical and Observed Responses in Experiment 3

\begin{tabular}{|c|c|c|c|c|c|c|c|c|c|c|}
\hline \multirow[b]{2}{*}{ Set Relation } & \multicolumn{5}{|c|}{ Correct Responses } & \multicolumn{5}{|c|}{ Observed Responses } \\
\hline & EQ & SB & SP & ov & DJ & EQ & SB & SP & ov & DJ \\
\hline EQ-SB & 0 & 21 & 0 & 0 & 0 & 5 & 17 & 4 & 6 & 4 \\
\hline SB-SB & 0 & 21 & 0 & 0 & 0 & 5 & 16 & 3 & 10 & 7 \\
\hline SB-SP & 21 & 21 & 21 & 21 & 21 & 11 & 12 & 9 & 14 & 12 \\
\hline SB-OV & 0 & 21 & 0 & 21 & 21 & 1 & 9 & 4 & 14 & 10 \\
\hline SP-SB & 21 & 21 & 21 & 21 & 0 & 12 & 13 & 11 & 19 & 6 \\
\hline SP-DJ & 0 & 0 & 21 & 21 & 21 & 8 & 6 & 10 & 17 & 11 \\
\hline OV-SP & 0 & 0 & 21 & 21 & 21 & 5 & 6 & 8 & 18 & 11 \\
\hline OVOV & 21 & 21 & 21 & 21 & 21 & 9 & 8 & 10 & 16 & 9 \\
\hline OV-DJ & 0 & 0 & 21 & 21 & 21 & 7 & 6 & 11 & 16 & 10 \\
\hline DJ-DJ & 21 & 21 & 21 & 21 & 21 & 14 & 13 & 11 & 16 & 18 \\
\hline
\end{tabular}

Note-The term on the left side of the set-relation pair (e.g., EQ in $E Q-S B$ ) refers to the set relation present in the first premise of the modified syllogism, and the term on the right side refers to the set relation present in the second premise. If Sets $A$ and $B$ appear in the first premise and Sets $B$ and $C$ appear in the second premise, then $S B$ in the first premise means $A$ is $a$ subset of $B$ and $S P$ in the first premise means $A$ is a superset of $B$, whereas $S B$ in the second premise means $B$ is a subset of $C$ and $S P$ in the second premise means $B$ is a superset of $C$. "Correct Responses" = number of subjects (of 21) that would have responded "true" if they had been logical; "Observed Responses" = number of subjects (of 21) that were observed to respond "true."

syllogism task. Similarly, one might expect that the order in which a pair of modified premises appears is not important (e.g., one might expect subjects to behave similarly on the modified premise pairs " $B$ and $C$ are disjoint sets: $\mathrm{A}$ and $\mathrm{B}$ are equivalent sets" and " $\mathrm{A}$ and B are equivalent sets; B and C are disjoint sets"). A test of this assumption was possible using the above data. The correlation between the number of "possibly true" responses to the derived set relations of the only two modified premise pairs in reverse order was very high $(r=.96)$. It was assumed that subjects performed logically on the remaining 10 pairs of set relations. Such an assumption is conservative if one expects the deductive strategy on modified syllogisms to explain behavior on standard syllogisms.

Given the above assumptions and the results of Experiment 3, one can predict the proportion of subjects in Experiment 2 that would indicate a particular set of combinations is possibly true or necessarily false for each subset of the 25 set relation pairs. These proportions were then used as the required probabilities in the deductive component.

\section{Discussion}

Not only do the deductive strategies appear to be similar in the modified syllogism task and the standard syllogism task, but, in addition, the use of alogical and incomplete deductive strategies appears to account for many of the incorrect responses that are not predicted by either premise misinterpretation or limits on information handling capacity. Both conclusions follow from the fact that the deductive model explains $74 \%$ of the variance and the limited-capacity model explains $60 \%$ of the variance. In short, when one controls for the effects of misinterpretation and limited information handling capabilities, the influence of alogical and incomplete deductive strategies remains strong.
The finding that subjects do not always derive all the logically possible set relations in a modified syllogism task such as Experiment 3 requires some discussion. One possible explanation of this finding is quite similar in form to the explanation of the limited-capacity component. Specifically, subjects may be more likely to derive one set relation than two set relations, more likely to derive two set relations than three set relations, and so on. The demands of the task and the limited motivation of subjects to do well are just two of the many possible reasons one might expect "progressively incomplete" deductive strategies. Such an explanation predicts that subjects are most likely to be correct when only one set relation can be derived from the modified syllogism and least likely to be correct when all five set relations can be derived from the modified syllogism (note that this is not the prediction one would make if chance were the sole determinant of behavior). The results are in good (but not perfect) agreement with the predictions. Recall that for one, three, four, and five logically possible derived set relations, the subjects were correct, respectively, $76 \%, 62 \%, 67 \%$, and $48 \%$ of the time. Note that the third percentage (i.e., the case for four logically possible, derived set relations) is based on the data from only one modified syllogism.

The above explanation of subjects' performance is similar to an explanation put forward by Ceraso and Provitera (1971). They, too, suggest that subjects may be using a progressively incomplete deductive strategy. However, because of the particular design that they employed, Ceraso and Provitera could not distinguish between the progressively incomplete explanation of deductive strategies and several alternative explanations. In the present case, Experiment 3 gives firm support to the notion of a progressively incomplete deductive strategy.

When more than one set relation can be derived 
from a modified syllogism, the subject can choose which set relation to respond to first. The findings from Experiment 3 suggest that, in those cases in which an overlap set relation is a logically possible conclusion, it will be the first conclusion considered. (Note that the overlap set relation is a logically possible conclusion for 8 of the 10 modified syllogisms.) Seventy-nine percent of the responses to the overlap set relation were correct. This compares with only $64 \%$ correct responses overall. Subjects are not simply being indiscriminate in their choice of the overlap set relation. When the overlap set relation does not follow logically from the modified syllogism, it is derived only $38 \%$ of the time (an error rate almost identical to the overall error rate).

The fact that subjects choose the overlap set relation so much of the time suggests either that subjects find it easier to derive the overlap set relation or that subjects find the overlap set relation in some sense more "believable" than other set relations they may have derived (in which case, they indicate that only the overlap set relation is logically possible, even though they may have derived other set relations). The distinction between these two explanations is important to an understanding of how subjects actually generate answers to the modified syllogisms. Future, more detailed studies of modified syllogism behavior will presumably want to separate these two explanations. Fortunately, the distinction does not affect the use of the modified syllogisms in an attempt to predict behavior on standard syllogisms.

Finally, it should be emphasized that the increase in the performance of the deductive model is not inevitable. Specifically, the parameters of the deductive model used to predict performance in the standard syllogism task (Experiment 2) were taken directly from altogether different studies (i.e., from Experiment 3 and from Sternberg \& Turner, Note 2). The exact model can be written as

$$
\begin{aligned}
Y_{i j k}= & {[1 / n] \sum_{r=1}^{n}\left[p_{1}\left(X_{i j k 1 r}\right)+p_{2}\left(X_{i j k 2 r}\right)+\right.} \\
& \left.p_{3}\left(X_{i j k 3 r}\right)+p_{4}\left(X_{i j k 4 r}\right)\right]+\epsilon,
\end{aligned}
$$

where $n$ is the number of subjects in Experiment 3 and $\mathrm{X}_{\mathrm{ijkmr} \mathrm{r}}$ is the probability that the ith subject in Experiment 2 would respond "possibly true" to the jth conclusion of the kth syllogism when the ith subject's interpretation of the premises are used (from Experiment 1), when $m$ set relation pairs are formed, and when the derived set relations of the rth subject in Experiment 3 are employed. As usual, $Y_{\mathrm{ijk}}$ represents the observed response of the ith subject in Experiment 2 to the jth conclusion of the $k$ th syllogism.

\section{EXPERIMENT 4}

The above models incorporate factors that appear to be important determinants of subjects' behavior in a "possibly true" syllogism task (i.e., a task that includes two responses, "possibly true" and "necessarily false"). The response "necessarily true" was not included for reasons mentioned earlier in the paper. However, the failure to include this response means that the results cannot be generalized to tasks that include "necessarily true" as one alternative response. Specifically, there is no way on a priori grounds to determine whether the above factors would exhibit the same relative influence in a "necessarily true" syllogism task as they do in a task that includes only the responses "possibly true" and "necessarily false." Thus, any comparisons of the results of this study with other studies is somewhat suspect since other studies have included the response "necessarily true." Accordingly, a fourth experiment was run that did include "necessarily true" as a response (as well as "possibly true or possibly false," and "necessarily false").

It should be noted that the four models developed in this paper can be extended in a straightforward manner to a "necessarily true" syllogism task. In particular, the readout stage of each model must now determine whether a particular conclusion is necessarily true, possibly true or false, or necessarily false, given a list of derived set relations.

\section{Method}

Twenty-two undergraduates at the University of Michigan participated in the fourth experiment. Their participation satisfied an introductory psychology course requirement.

In the first part of the experiment, each subject received four premises: "All Sats are Kuls," "No Sats are Kuls," "Some Sats are Kuls," and "Some Sats are not Kuls." Underneath each premise appeared five combinations or derived set relations: "All Sats are Kuls and all Kuls are Sats," "All Sats are Kuls and at least one Kul is not a Sat," "All Kuls are Sats and at least one Sat is not a Kul," "At least one Sat is a Kul, at least one Sat is not a Kul, and at least one Kul is not a Sat," and "No Sats are Kuls and No Kuls are Sats." Subjects were asked to indicate whether the combination was necessarily true, possibly true or false, or necessarily false. Each subject received a different ordering of the premises and combinations.

In the second part of the experiment, each subject received 32 syllogisms. Each syllogism was followed by four conclusions (see Experiment 2). Subjects were asked to indicate whether each conclusion was necessarily true, possibly true or false, or necessarily false. Every succeeding pair of subjects saw the full complement of 64 syllogisms. The 32 syllogisms for one member of a pair were selected randomly from the 64 possible syllogisms. The syllogisms were then presented randomly to each subject. The order of the conclusions was different for every subject. Subjects in both this part of the experiment and in the aforementioned part were allowed as much time as they needed to finish the experiment.

\section{Results}

Three subjects were not included in the analysis because they gave exactly the same pattern of responses to each of the 32 syllogisms. Discussion with these subjects indicated a misunderstanding of the task.

Overall, subjects in the first part of the experiment interpreted $29 \%$ of the 20 derived set relations from the four premises in a strictly logical fashion. In order to compare the results of this experiment with the results 
from Experiment 1, it is necessary to ignore the distinction between a "necessarily true" response and a "possibly true" or "possibly false" response. If this distinction is ignored, then one finds that $41 \%$ of the interpretations were correct (still substantially below the $60 \%$ figure in Experiment 1). An examination of the individual premises indicates that subjects' performance in Experiment 4 was better than subjects' performance in Experiment 1 on only one of the premises: "All C are A." Finally, it should be noted that the Chapman and Chapman (1959) model agrees with a greater percentage of subjects' interpretations of the premises than do the four other premise misinterpretation models.

The logical model explains 37\% of the variance from the syllogism task in the second part of Experiment 4. The premise model explains $52 \%$ of the variance. And the limited-capacity and deductive models both explain $70 \%$ of the variance.

\section{Discussion}

The results from the analyses using the logical, premise, and limited-capacity models in a "necessarily true" syllogism task essentially parallel the results from the earlier "possible true" syllogism task (i.e., the increases in the variance explained from model to model are quite similar in the two tasks). However, the deductive model shows no advantage over the limited-capacity model in the "necessarily true" syllogism task (i.e., Experiment 4), whereas it does show a decided advantage in the "possibly true" syllogism task (i.e., Experiment 2).

It should be noted at the outset that the above results are not consistent with a model that assumes that subjects are using logical deductive strategies. If subjects were using logical deductive strategies, then the limitedcapacity model should have outperformed the deductive model, since (1) the limited-capacity model assumes logical deductive strategies, (2) the deductive model assumes alogical and incomplete deductive strategies, (3) the models differ on only the deductive component, and (4) the models make different predictions. It remains to show what models are consistent with the above results (i.e., the finding that the deductive model interacts with the task demands).

There are many different reasons why the predictive power of the deductive model might vary from task to task. Consider the possibility that factors in Experiment 4 mask the influence of the deductive component. Specifically, consider the possibility that subjects in both Experiments 2 and 4 have alogical and incomplete deductive strategies but that subjects in Experiment 4 are less likely than subjects in Experiment 2 to respond "necessarily false." Note that it is the "necessarily false" responses that are of most interest, since it is a preponderance of these responses that distinguishes the deductive model from other models (i.e., the existence of incomplete deductive strategies leads to the failure to derive certain set relations that are logically possible; this in turn can lead the subject to indicate that a conclusion is never possible, or necessarily false, when in fact the conclusion is logically possible).

The fact that there exist different response categories in Experiments 2 and 4 suggests that a change in the number of "necessarily false" responses can potentially be traced to the use of the different categories of response. In particular, the change might be explained as follows. Note that, in general, subjects may be uncomfortable when their reasoning requires them to label a conclusion as necessarily false. The bias against accepting a nonpropositional conclusion (i.e., the tendency to label conclusions as necessarily true that are not so) in some syllogism tasks supports the above notion (e.g., see Chapman \& Chapman, 1959). Since there are only two response categories in Experiment 2, subjects may divide their responses between "possibly true" and "necessarily false" in those situations in which they have initially found that a conclusion is necessarily false (presumably, the more sure subjects are that a conclusion must necessarily be false, the less likely they are to divide their response). Since there are three response categories in Experiment 4, the subject may again divide his or her responses among the different categories. However, note that this implies that there will be a $33 \%$ decrease in the number of "necessarily false" responses in Experiment 4. Thus, the number of response categories could influence the number of "necessarily false" responses.

If the above explanation were true, then there should be a shift away from "necessarily false" responses in Experiment 4. A subset of the syllogisms offers a relatively pure test of the shift. In particular, consider the four figures of the syllogism with a universal, negative quantifier in the first and second premises (e.g." "No $A$ are B" and "No B are C"). A response of "necessarily false" to one or more of the conclusions is consistent with an incomplete deductive strategy but is left unexplained by either premise misinterpretation or limited-capacity components. Thus, the four figures of the aforementioned syllogism provide a good test of the proposed shift, since behavior on these syllogisms is influenced by variations in the deductive strategy alone.

It will be recalled that many of the conclusions to the above four syllogisms in Experiment 2 were marked "necessarily false" (approximately 28\%). However, note that relatively few conclusions are marked "necessarily false" in Experiment 4 (approximately 10\%). Thus, there is a shift away from the "necessarily false" responses in the predicted direction.

The above explanation is not the only possible explanation for the failure to find an advantage of the deductive model over the limited-capacity model in Experiment 4. For example, subjects might use entirely different deductive strategies in a "necessarily true" syllogism task than they do in a "possibly true" syllogism task (although what these strategies might be is not altogether clear). The above would imply that behavior on the isolable stage (i.e., the deductive stage when 
studied in isolation) does not mimic behavior on the same stage embedded in a more complex task. The various explanations need to be more rigorousiy tested in future studies.

In short, the premise misinterpretation and limitedcapacity components have similar effects in the "necessarily true" and "possibly true" syllogism tasks. However, the influence of the deductive component may interact with the nature of the task. It was argued that the existence of alogical and complete deductive strategies is consistent with the findings in Experiment 4 if one assumes a shift in the response probabilities away from "necessarily false." Evidence was introduced that pointed to such a shift.

\section{GENERAL DISCUSSION}

While the above models appear to have achieved some measure of predictive success, there are important limitations to the models that should be emphasized. First, note that the premise, limited-capacity, and deductive components may not explain the same relative proportions of variance in tasks less demanding than the ones reported in this paper. Specifically, the tasks reported in this paper require subjects to be explicit about aspects of the syllogistic reasoning process that might in other situations be ignored. For example, consider a task in which subjects indicate which, if any, conclusions were necessarily true. Subjects in this task could adopt the relatively straightforward (and incorrect) strategy suggested by Woodworth and Sells (1935), whereas subjects in the above experiments could not adopt such a simple strategy.

Second, the models reported in this paper assume that subjects are not affected by the substantive content of the premises. However, as mentioned in the introduction, this assumption receives little, if any, support. Therefore, the models would have to be modified were the premises given something other than a neutral substantive content.

Finally, the models proposed in this paper may not account (as is) for all the various effects that have been found in syllogism tasks using premises with a content similar to that employed in the above experiments (i.e., a neutral substantive content). However, it should be noted that the models have been able to account for at least some portion of each of the effects that have been examined to date. For example, it will be recalled that several investigators have found that the figure of the syllogism has a large influence on subjects' behavior (Dickstein, 1978a; Frase, 1968; Johnson-Laird \& Steedman, 1978; Roberge, 1971). Fisher (Note 4) has shown that misinterpretation can explain some (but not all) of the observed figural effects.

A second, and perhaps more instructive, example follows from recent work by Dickstein (1978b). Dickstein argues that there are various types of errorladen processes that control behavior on a select subset of 32 syllogisms. The subset of syllogisms is divided into three groups. Based on the nature of the error processes specific to a group, Dickstein is able to predict the relative difficulty of the syllogisms in the group (the difficulty was measured as percentage correct). Dickstein's predictions were confirmed, and thus the explanations of the errors receive some support.

It was possible to perform much the same analysis in this study. The results of the analysis agree with the findings of Dickstein (1978b); that is, the relative difficulty among the three groups of syllogisms remained the same. In the present case, it was possible to perform an additional analysis. Specifically, it was possible to determine the variance explained in the three groups using the logical and deductive models. Not surprisingly, the variance explained by the logical model decreased markedly from the easiest to the most difficult group (this simply reflects the decrease in the percentage correct). However, the variance explained by the deductive model for each of the three groups remained almost identical and very high from group to group. In other words, the relative difficulty among the three groups of syllogisms singled out by Dickstein appears to be explained by the factors that are incorporated in the deductive model, since, after controlling for these factors, the difficulty of the three groups is roughly identical. (Note that Dickstein's explanations of errors overlap with the explanations of errors proposed in this paper; thus the fact that the deductive model accounts for the errors is not necessarily at odds with Dickstein's model.)

In short, the models proposed in this paper have a limited generality. However, if the models are applied to a relatively restricted domain of syllogism tasks (i.e., tasks with neutral premises), then the models can be shown to achieve a moderate measure of success. The models explain a fair portion of the variance. In addition, the models can account (with varying degrees of success) for certain of the effects reported in other studies.

\section{CONCLUSION}

The present study focused on those factors that influence subjects' behavior in a syllogistic reasoning task. At least three factors appear to play an important role: the interpretation of the premises, the limited capacity of short-term memory, and the deductive strategy. The study indicates that much can be learned about a complex task such as syllogistic reasoning from the analysis of individual factors or components in isolation. The study suggests ways in which one can estimate the relative importance of a proposed factor. And the study suggests that various current explanations of errors are the consequence of complex interactions among the components of the syllogistic reasoning process. This last finding strongly suggests that future studies of syllogistic reasoning should not confine 
themselves to the study of simple effects unless the effects are incorporated as components of a more complex model.

\section{REFERENCE NOTES}

1. Guyote, M. J., \& Sternberg, R. J. A transitive-chain theory of syllogistic reasoning (ONR Tech. Rep. 5). New Haven, Conn: Department of Psychology, Yale University, April 1978.

2. Sternberg, R. J., \& Turner, M. E. Components of syllogistic reasoning (ONR Tech. Rep. 6). New Haven, Conn: Department of Psychology, Yale University, April 1978.

3. Erickson, J. R., Wells, G. L., \& Traub, B. H. Tests of a model of formal syllogistic reasoning. Paper presented at the annual meeting of the Psychonomic Society, Boston, November 1974.

4. Fisher, D. L. Syllogistic reasoning: Computer models and individual differences. Unpublished manuscript, 1979. (Available from D. L. Fisher, K208 Lloyd Hall, 580 Union Drive, University of Michigan, Ann Arbor, Michigan 48109).

\section{REFERENCES}

Ceraso, J., \& Provitera, A. Sources of error in syllogistic reasoning. Cognitive Psychology, 1971, 2, 400-410.

Chapman, L. J., \& Chapman, J. P. Atmosphere effect re-examined. Journal of Experimental Psychology, 1959, 58, 220-226.

Dickstein, L. S. Effects of instructions and premise order on errors in syllogistic reasoning. Journal of Experimental Psychology: Human Learning and Memory, 1975, 1, 376-384.

Dickste in, L. S. Differential difficulty of categorical syllogisms. Bulletin of the Psychonomic Society, 1976, 8, 330-332.

Dickstein, L. S. The effect of figure on syllogistic reasoning. Memory \& Cognition, 1978, 6, 76-83. (a)
Dickstein, L. S. Error processes in syllogistic reasoning. Memory \& Cognition, 1978, 6, 537-543. (b)

Erickson, J. R. A set analysis theory of behavior in formal syllogistic reasoning tasks. In R. Solso (Ed.), Theories of cognitive psychology: The Loyola symposium (Vol. 2). Hillsdale, N.J: Erlbaum, 1974.

Erickson, J. R. Research on syllogistic reasoning. In R. Revlin \& R. E. Mayer (Eds.), Human reasoning. Washington, D.C: Winston, 1978.

Frase, L. T. Associative factors in syllogistic reasoning. Journal of Experimental Psychology, 1968, 76, 407-412.

Johnson-Latrd, P. N., \& Steedman, M. The psychology of syllogisms. Cognitive Psychology, 1978, 10, 64-99.

Revlin, R., \& Leiner, V. O. The effect of personal biases on syllogistic reasoning: Rational decisions from personalized representations. In R. Revlin \& R. E. Mayer (Eds.), Human Reasoning. Washington, D.C: Winston, 1978.

REvL1s, R. Syllogistic reasoning: Logical decisions from a complex data base. In R. Falmagne (Ed.), Reasoning: Representation and process. Hillsdale, N.J: Erlbaum, 1975. (a)

REvuIs, R. Two models of syllogistic reasoning: Feature selection and conversion. Journal of Verbal Learning and Verbal Behavior, 1975, 14, 180-195. (b)

Roberge, J. Further examination of mediated associations in deductive reasoning. Journal of Experimental Psychology, 1971, 87, 127-129.

Wilkins, M. The effect of changed material on ability to do formal syllogistic reasoning. Archives of Psychology, 1928, $16,83$.

Woodworth, R. S., \& Sells, S. B. An atmosphere effect in formal syllogistic reasoning. Journal of Experimental Psychology, 1935, 18, 451-460.

(Received for publication December 22, 1980; revision accepted April 10, 1981.) 